\title{
Research on the Application of Automatic Control System in Mechanical Engineering
}

\author{
Kaihong Xie \\ Yunnan Economics Trade and Foreign Affairs College, Kunming, Yunnan, 650114
}

Keywords: Mechanical Engineering; Automatic Control Systems; Sensors

\begin{abstract}
With the continuous innovation and development of science and technology in our country, the automation control system has gradually infiltrated into various industries in the society. The automation control system plays a very important role in the modern mechanical engineering. In this paper, the mechanical engineering automatic control system in the use of the issue of analysis and research, mainly for the development of both to be an introduction, and analysis of Chinese current state of the art of automatic control systems and application analysis.
\end{abstract}

\section{Introduction}

As society continues to develop and its economy continues to grow, the daily work of human beings has gradually become more and more advanced. As an important support for the development of the productive forces, laborers have done a great deal of work instead of laboring. This shows that in the fields of human production, the status of labor appliances in life is very important. And with the gradual development of wood machinery to the late metal structure, the production process of labor appliances has also achieved a qualitative change, which is the basis for the development of modern mechanical engineering. However, with the rapid development of automation technology and computer technology, mechanical engineering has made further development and gradually realized the automatic control. We must be proficient in using automated control systems to continually adapt to increasingly complex production processes while increasing productivity.

\section{The Important Role of Mechanical Engineering in Reality}

In the process of human production and living are inseparable from the use of labor appliances, first of all human beings in order to transform the natural transformation of life is to use the manual to complete the work, but after all, manpower is limited and can not face all the work. With the increasing demand, people rely more and more on their manual work more and more restrictions, based on this mechanical apparatus came into being, human beings constantly improve it, many times the machinery can be completed with the hands of people can not match the work, but also to ensure the quality and efficiency. With the continuous innovation and development of technology, people have accumulated a great deal of valuable experience in using machinery, and the research on machinery has been further deepened. In today's society, mechanical engineering has gradually entered the high-tech, both in terms of quality, precision and other contemporary mechanical engineering have achieved great results. For example, in agriculture, construction, military, medical, exploration and many other fields, are inseparable from the use of mechanical engineering.

\section{The Development Status Quo of Chinese Automation and Control System}

Modern mechanical engineering has a unique automatic control system, through this system for automatic control of the entire mechanical engineering. The automatic control system is the use of a variety of high-tech, unmanned operation to allow mechanical engineering to automatically work to complete the production work, or to people in accordance with pre-set steps automatically completed operation. Under normal circumstances, the automation and control system includes a 
controller (such as PLC), actuators, sensors, controlled objects (such as motor) composed of four parts.

The development of automatic control system is an inevitable result of economic development level up to a certain stage. Since the 21st century, the application of automatic control system in mechanical engineering has received more attention and attention from all parties. In the early days, automatic control was generally based on a mechanical device area, and the commands were now mainly executed by a microcontroller or a PLC controller. With the constant innovation and development of science and technology, the direction of intelligent development is a new trend of automatic control system. In the future, more and more high-tech technologies such as optoelectronic computers and nano-optoelectronics will make the whole system more stable and reliable, the system response time will be shorter, Higher accuracy.

After the 1940s, with the generation of classical control theory, most of the input signals are analog signals, and the switch signals are output after being processed by the control system. As the demand for multivariate becomes more and more, the actual production process also occurs for more realistic data, we need to get the desired control process by fast computing data, and the rapid development of computer technology has laid a good foundation for this problem. With the development of science and technology, the control of mechanical engineering has also become more and more complex. In the actual production process, many non-linear technical problems are often encountered and the corresponding mathematical models can not be established to solve. Therefore, the automatic control Difficult, but the effect is not very satisfactory. The birth of intelligent control allows this technical problem to be solved, and the development of a very wide range of space will be the future of new trends in the development of automatic control [1].

\section{The Main Research Content of Automatic Control System}

The infrastructure to automate control functions is the computer because of the wide range of mechanical engineering involved and many industries need automatic control systems that fit their characteristics. Therefore, it should be based on the controlled object's function, parameters, uses and other characteristics of their own choice of computer. Under normal circumstances, if the controlled object is relatively simple and the amount of analog and switch is small, the control logic of the control system is generally relatively simple, often using PLC controller or microcontroller to achieve control functions, according to the actual control logic Write a program to achieve control. If the accused object is more complex, large-scale or need to deal with the information is very large, it must be used IPC; to the system bus as the basis for the structure, the way to communicate digital control of the control template and the computer directly to the data. If the controlled mechanical engineering is more complex and the control system has more levels, we can consider using the distributed automatic control system to analyze the characteristics of the control objects in each layer separately. According to the actual situation, choose PLC bus, single chip microcomputer, or miniature Computer, etc. to achieve different functions. And according to the actual situation to develop different control programs, and then comprehensive comparison and finally determine the most appropriate control program.

The key support for the operation of the automatic control system is the control software. There are mainly two major categories of application software. On the one hand, in order to meet various control requirements, the control application software provided mainly by the requirements of the optoelectronic system. MATLAB software can be used to carry out analysis and research, you can improve the quality of the system to complete the performance indicators. On the other hand is the system software, such as common Windows operating platform, which is mainly composed of driver, database, operating system, network connection and diagnosis system. The main computer is the appropriate scheduling and maintenance, is responsible for coordinating the computer hardware and software. With the constant innovation and development of technology, application software is constantly being standardized, with more complete functions and more complete parameters.

Human-machine interface is a key part of automatic control, which is an indispensable condition for the development of automatic control system. People occupy the leading position in this process, 
and are responsible for the research and development of the whole system. In the design of the system hardware and software should consider the safety while paying attention to the convenience of operation, simple. Through a reasonable design, the system can meet all operational requirements, while ensuring the ultimate decision-making function of people, focusing on the development of new ideas of human nature. Reasonable design of information interfaces, physical interfaces and other related technical interfaces to facilitate all aspects of the connection between each other. In terms of information processing, the intelligent interface has a very big advantage. It is an important point of current research. The man-machine interface is also an important part of the automatic control system.

A digital, serial, multipoint communication data bus is installed between the field device and the automation unit. Such as common typical data bus: Controller Area Network HART, CAN, Fieldbus FF and so on. For example, the CAN bus is widely used in the field of machinery manufacturing, with a very high data transfer rate, and anti-electromagnetic interference ability, the detection rate for the error is also very high, to smooth the realization of real-time automatic control. However, there are still some problems in the transmission distance of the CAN bus currently studied.

\section{The Research Results of Chinese Automatic Control System}

The application of the sensor in modern mechanical engineering plays a very important role because the sensor is very important for the overall control effect and closely related to the later operation. Therefore, the performance of the sensor must be guaranteed in line with the requirements of the design, so as to improve the level of automatic control. On the one hand, the quality of the sensor itself should be protected, for example, subject to greater external interference, etc., will not be damaged, affecting the accuracy of the data detected, it should have some anti-interference ability, anti-pollution ability, but also temperature to adapt to a wider range; the other hand, the sensor should also have the ability to accurately read the information, to quickly obtain effective information. However, the current situation in our country is that many sensors can only realize the above functions in the laboratory. However, the actual use of the sensor is often not satisfactory due to various external factors and the performance is not fully exerted. Therefore, this is an important obstacle to the development of mechanical engineering automation and control.

Many mechanical engineering site construction environment is very harsh, on-site construction conditions are getting worse, so machinery in the field when the job often have a serious vibration, which directly affects the use of results. For example, in the power source of the hydraulic system by the impact of the outside world or the impact of load are made of mechanical use is not good. So mechanical tools should have very high shock resistance. On the other hand, such as on-site pollutants, water, gravel, etc. are likely to enter the site construction machinery, which also directly interfere with the normal operation of mechanical tools, in order to avoid such incidents should allow the system has a good sealing function, thus increasing the degree of protection of mechanical tools. At the same time mechanical engineering system should also have high anti-interference ability to avoid outside noise or the environment affect the normal operation of the system.

\section{Rotary Drilling Machine Automatic Control System in the Practical Application}

In the actual drilling process, the rotary drilling rig's mast must be vertical, otherwise the drilling effect is not very satisfactory. In order to tilt some timely rectification, automatic control system in this section will automatically detect the mast tilt, timely correction, let it restore the vertical state. Mainly through the biaxial tilt sensor to detect the inclination, and then input to the analog input module, after the PLC program logic conversion, the control signal is passed to the mast controller, the controller moment to control the mast to prevent its tilt [2].

In the process of construction, often encounter the car back and forth rotation, this time because of its load is relatively large, there may be positioning problems can not be accurately drilling positioning, it is necessary to establish an automatic rotary positioning control system. This will come in to reduce the ability to loss, and the positioning is very accurate and there is almost no big 
error, torque is also within the prescribed range. Automatic rotary positioning control system is built on the electro-hydraulic proportional control technology, CAN-BUS control bus and signal feedback control technology based on. Mainly including the man-machine interface, the controller, the encoder, the operation knob, in the man-machine contact surface has the concrete hole hypothesis input box, according to the actual situation hypothesis value, when the operation knob hits the automatic time, the encoder can detect the location information in time, and then through the PLC processing, and finally the control signal to the actuator, making the drill can be automatically positioned.

In actual work, during the earth returning process, if the drill does not stop in time when contacting the ground, and if the hoist still continues to work, it may cause the rope to be chaos easily while being lowered. If the rope subjected to excessive pressure, if you do not stop winding hoisting work may result in serious accidents. Therefore, automatic stop of the control system is very important for the safety of mechanical engineering.

In summary, in the process of human social development, mechanical engineering has played a very important role, with the continuous development of Chinese processing industry, manufacturing industry, its workload is also increasing, while the quality requirements are gradually Therefore, we must make full use of the automatic control system. Because only by making full use of automatic control system can effectively improve the overall work efficiency, and this is the key to the development of mechanical and electrical integration. With the development of science and technology, the future development of automation and control technology will move toward the direction of artificial intelligence.

\section{References}

[1] Du Zhong, Tang Yong. Research and Application of Automatic Control System in Modern Mechanical Engineering [J]. Journal of Hunan Industry Polytechnic, 2013 (5): 24-26.

[2] Ding Meiling. Discussion on the application of automatic control system in modern mechanical engineering [J]. Science and Technology Information, 2014,12 (16): 55-57. 